\title{
The Wnt signaling pathway and mitotic regulators in the initiation and evolution of mantle cell lymphoma: Gene expression analysis
}

\author{
YOSHIZO KIMURA ${ }^{1}$, FUMIKO ARAKAWA ${ }^{1}$, JUNICHI KIYASU ${ }^{1}$, HIROAKI MIYOSHI ${ }^{1}$, \\ MAKI YOSHIDA $^{1}$, AYAKO ICHIKAWA ${ }^{1}$, DAISUKE NIINO ${ }^{1}$, YASUO SUGITA ${ }^{1}$, TAKASHI OKAMURA ${ }^{2}$, \\ ATUSHI DOI $^{3}$, KAORI YASUDA ${ }^{3}$, KOSUKE TASHIRO $^{4}$, SATORU KUHARA ${ }^{4}$ and KOICHI OHSHIMA ${ }^{1}$ \\ Departments of ${ }^{1}$ Pathology and ${ }^{2}$ Hematology, School of Medicine, Kurume University, Kurume, Fukuoka; \\ ${ }^{3}$ Cell Innovator Inc., Venture Business Laboratory of Kyushu University; ${ }^{4}$ Department of Bioscience and Biotechnology, \\ Faculty of Agriculture, Kyushu University, Fukuoka, Japan
}

Received April 10, 2013; Accepted May 20, 2013

DOI: $10.3892 /$ ijo.2013.1982

\begin{abstract}
For an accurate understanding of mantle cell lymphoma (MCL), molecular behavior could be staged into two major events: lymphomagenesis with the $\mathrm{t}(11 ; 14)$ translocation (initiation), and evolution into a more aggressive form (transformation). Unfortunately, it is still unknown which genes contribute to each event. In this study, we performed cDNA microarray experiments designed based on the concept that morphologically heterogeneous MCL samples would provide insights into the role of aberrant gene expression for both events. A total of 15 MCLs were collected from the files, which include a total of $237 \mathrm{MCL}$ patients confirmed by histology as CCND1-positive. We posited four stepwise morphological grades for MCL: MCL in situ, MCL with classical form (cMCL), MCL with aggressive form (aMCL), and MCL with intermediate morphology between classical and aggressive forms at the same site (iMCL). To identify genes involved in initiation, we compared the tumor cells of MCL in situ $(\mathrm{n}=4)$ with normal mantle zone B lymphocytes $(n=4)$, which were selected by laser microdissection (LMD). To identify genes contributing to transformation, we selected the overlapping genes differentially expressed between both cMCL $(n=4)$ vs. aMCL $(n=5)$ and classical vs. aggressive areas in iMCL $(n=2)$ obtained by LMD. A significant number of genes $(n=23, p=0.016)$ belonging to the Wnt signaling pathway were differentially expressed in initiation. This specific activation was confirmed by immunohistochemistry, as MCL in situ had nuclear localization of phosphorylated-
\end{abstract}

Correspondence to: Dr Yoshizo Kimura, Department of Pathology, School of Medicine, Kurume University, Asahi-machi 67, Kurume, Fukuoka 830-0011, Japan

E-mail: kimura_yoshizou@kurume-u.ac.jp

Key words: mantle cell lymphoma, initiation, transformation, cDNA microarray analysis, laser microdissection, Wnt signaling pathway, mitotic regulator $\beta$-catenin with high levels of cytoplasmic Wnt3 staining. For transformation, identified 60 overlapping genes included a number of members of the $p 53$ interaction network $(C D C 2$, BIRC5 and FOXM1), which is known to mediate cell cycle progression during the G2/M transition. Thus, we observe that the Wnt signaling pathway may play an important role in initial lymphomagenesis in addition to $t(11 ; 14)$ translocations, and that specific mitotic regulators facilitate transformation into more aggressive forms.

\section{Introduction}

The pathological category of low-grade B cell non-Hodgkin's lymphoma (NHL) encompasses mature B cell neoplasms with small to medium-sized cells such as follicular lymphoma (FL), marginal zone lymphoma (MZL), B cell chronic lymphocytic leukemia/small lymphocytic lymphoma (B-CLL/SLL), and mantle cell lymphoma (MCL). The histological transformation of low-grade lymphomas is thought to have a major impact on their prognosis, however, the clonal relationship between the two neoplasm types and the pathogenesis underlying the progression of the disease are still controversial.

MCL is considered a well categorized B cell NHL because virtually all cases feature the chromosomal translocation $\mathrm{t}(11 ; 14)(\mathrm{q} 13 ; \mathrm{q} 32)(\operatorname{IgH} / C C N D 1)$ which leads to cyclin D1 (CCND1) overexpression. Despite this strong genetic hallmark, MCL presents in a broad spectrum of morphological forms with a high number of additional chromosomal abnormalities. From a practical point of view, we can detect the mantle zone growth pattern, which represents the initial component of MCL, in only $8.6 \%$ of cases (1). Aggressive transformation in MCL is more notable, occurring in $32 \%$ of living cases and in $70 \%$ of autopsy cases (2). Hence, the presence of pathological evidence such as characteristic cytologies (small, classical, blastic and pleomorphic) or proliferating patterns (mantlezone, nodular and diffuse) at the time of biopsy seems to depend on temporal changes in the accumulation of molecular genetic alterations.

For an accurate understanding of MCL, there are some questions requiring resolution. First, why is this lymphoma 
Table I. Data of studied patients.

\begin{tabular}{|c|c|c|c|c|c|c|c|c|}
\hline Case & Age & Sex & Diagnosis & Tissue & Growth pattern & PI (Ki-67) & IgH/CCND1 & RNA extraction \\
\hline $\mathrm{R} 1$ & 36 & $\mathrm{~F}$ & Benign lymphadenitis & $\mathrm{LN}$ & - & ND & ND & LMD \\
\hline $\mathrm{R} 2$ & 25 & $\mathrm{~F}$ & Benign lymphadenitis & $\mathrm{LN}$ & - & ND & ND & LMD \\
\hline $\mathrm{R} 3$ & 54 & M & Benign lymphadenitis & $\mathrm{LN}$ & - & ND & ND & LMD \\
\hline R4 & 14 & M & Benign lymphadenitis & $\mathrm{LN}$ & - & ND & ND & LMD \\
\hline MCL112 & 68 & M & MCL in situ & $\mathrm{LN}$ & $\mathrm{MZ}$ & 15 & + & LMD \\
\hline MCL113 & 49 & M & MCL in situ & $\mathrm{LN}$ & $\mathrm{MZ}$ & 10 & + & LMD \\
\hline MCL114 & 73 & M & MCL in situ & $\mathrm{LN}$ & $\mathrm{MZ}$ & 20 & + & LMD \\
\hline MCL71 & 57 & M & MCL in situ & $\mathrm{LN}$ & $\mathrm{MZ}$ & 5 & + & LMD \\
\hline MCL126 & 79 & M & Classical MCL (cMCL) & $\mathrm{LN}$ & $\mathrm{N}$ & 25 & + & Whole \\
\hline MCL135 & 59 & M & $\mathrm{cMCL}$ & $\mathrm{LN}$ & $\mathrm{N}$ & 20 & + & Whole \\
\hline MCL141 & 75 & M & cMCL & $\mathrm{LN}$ & $\mathrm{N}$ & 25 & + & Whole \\
\hline MCL200 & 75 & M & cMCL & $\mathrm{LN}$ & $\mathrm{N}$ & 30 & + & Whole \\
\hline MCL80 & 80 & M & Intermediate MCL (iMCL) & $\mathrm{LN}$ & N\&D & $20 / 80$ & + & LMD \\
\hline MCL132 & 67 & $\mathrm{~F}$ & iMCL & $\mathrm{LN}$ & N\&D & $15 / 95$ & + & LMD \\
\hline MCL5 & 71 & M & Aggressive MCL (aMCL) & $\mathrm{LN}$ & $\mathrm{D}$ & 70 & + & Whole \\
\hline MCL40 & 77 & $\mathrm{~F}$ & aMCL & $\mathrm{LN}$ & $\mathrm{D}$ & 70 & + & Whole \\
\hline MCL98 & 87 & M & aMCL & LN & D & 95 & + & Whole \\
\hline MCL102 & 77 & M & aMCL & $\mathrm{LN}$ & $\mathrm{D}$ & 75 & + & Whole \\
\hline MCL107 & 76 & M & aMCL & $\mathrm{LN}$ & D & 60 & + & Whole \\
\hline
\end{tabular}

LMD, laser microdissection; PI, proliferation index; ND, not done; LN, lymph node; MZ, mantle zone; N, nodular; D, diffuse.

characterized by biological heterogeneity despite the characteristic translocation event? Second, what is required for the initial pathogenesis of MCL in addition to the $\mathrm{t}(11 ; 14)$ translocation? A recent study has shown that the $\mathrm{t}(11 ; 14)$ translocation alone is not sufficient to produce tumors (3) and, though at very low levels, $\mathrm{t}(11 ; 14)$-positive cells have been found in the blood of healthy individuals (4). Thus, key molecular behavior in MCL is thought to be divided into two major stages: initial lymphomagenesis in addition to the $\mathrm{t}(11 ; 14)$ translocation (initiation) and the accumulation of variable secondary genomic alterations occurring over time, leading to the evolution into more aggressive forms (transformation).

Recently, microarray profiling studies have identified differential expression of several genes in the progression of MCL (5-7). Some of these studies have used purified mantle zone B lymphocytes sorted from reactive tonsillectomy specimens for gene expression profiling to avoid contamination with stromal cells, $\mathrm{T}$ cells and macrophages $(6,7)$. In these studies, several genes identified as overexpressed in aggressive form are known to be involved in cell cycle control or apoptotic cell death, and several genes related to the PI3K/AKT, WNT and TGF- $\beta$ signaling pathways are reportedly important in the pathogenesis of MCL. Although morphological heterogeneity remains a central issue in the prognosis and treatment of this lymphoma, no gene expression profiling studies to date have attempted to identify the genes and signaling pathways involved in the each event separately. The present study was designed based on the concept that gene expression profiling of morphologically heterogeneous MCL samples would provide insight into the role of aberrant gene expression for both initial lymphomagenesis and transformation events.

\section{Materials and methods}

Patients and tissue samples. We performed cDNA microarray experiments using frozen tissues of 19 lymph node biopsies. A total of 15 MCLs were collected from the files of the Departments of Pathology of Kurume University (Fukuoka, Japan), which include a total of $237 \mathrm{MCL}$ patients (1) confirmed by histology as CCND1-positive (Table I). All 15 cases were subjected to cytogenetic and/or FISH studies and found positive for the I $g H / C C N D 1$. This study was approved by the Kurume University Institutional Review Board, and patients provided informed consent in accordance with the Declaration of Helsinki.

In order to identify differentially expressed genes and their contribution to each event, we posited four stepwise morphological grades for MCL: MCL in situ, MCL with classical form (cMCL), MCL with aggressive form (aMCL), and MCL with intermediate morphology between classical and aggressive forms at the same site (iMCL) (Fig. 1). Namely, MCL in situ is defined as those samples with a very thin neoplastic mantle zone growth pattern and very little or no spreading of tumor cells into interfollicular areas. cMCL is characterized by a prominent nodular proliferation of atypical medium-sized tumor cells (classical form) without diffuse proliferation. aMCL is designated as a combination of two morphological forms: blastoid and pleomorphic. iMCL contains distinct areas of both classical and aggressive forms.

For evaluation of initiation, we compared samples from the tumor cells of MCL in situ ( $\mathrm{n}=4)$ with those from normal mantle zone B-lymphocytes from benign lymphadenitis $(n=4)$. Samples were derived from selected specimens by means of laser microdissection (LMD). We hypothesize that transformation 


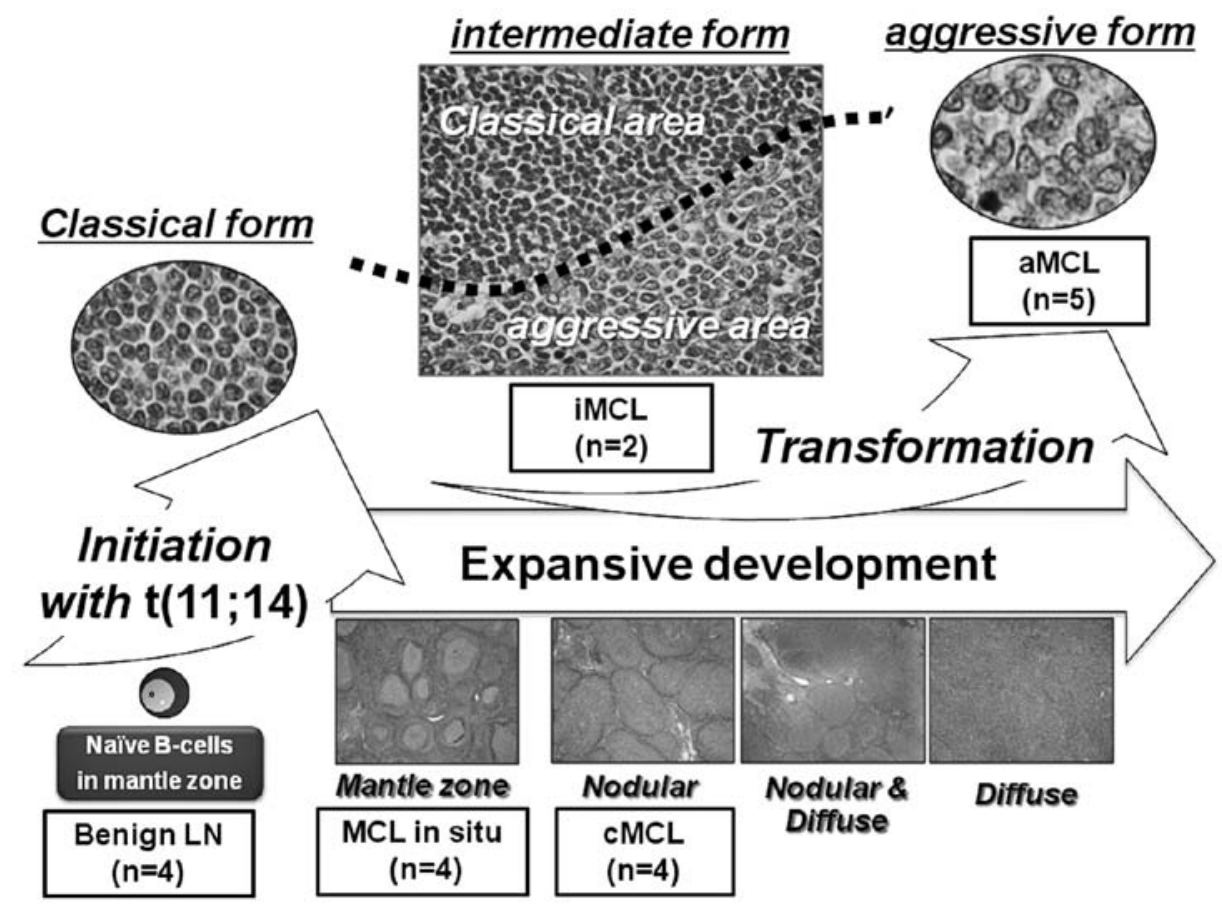

Figure 1. Schema of the stepwise morphological grades for MCL used in the present study. This diagram shows the morphological evolution of MCL occurring in parallel with transformational development (classical, intermediate, and aggressive) and expansive development (mantle zone, nodular, nodular \& diffuse, and diffuse). The origin of MCL cells is postulated as naive CD5-positive pre-germinal center B cells. In order to identify the genes contributing to each event, we posited four stepwise morphological grades for MCL: MCL in situ, MCL with classical form (cMCL), MCL with aggressive form (aMCL), and MCL with intermediate morphology between classical and aggressive forms at the same site (iMCL). MCL in situ represents the mantle zone growth pattern in that the neoplastic mantle zone was very thin and there was very little if any spreading of tumor cells into interfollicular areas. cMCL is characterized by a prominent nodular proliferation of atypical medium-sized tumor cells (classical form) without diffuse area. iMCL contains both classical and aggressive form areas but separated by border lines (black dotted line). aMCL was designated a combination of two morphological forms, blastoid and pleomorphic.

into aMCL is a multistep process, similar to the progression of carcinomas. It may be beneficial to subdivide aMCL neoplasms into two major subtypes: 'aMCL with genetic stepwise process' which results from the accumulation of many molecular genetic alterations and 'de novo aMCL' resulting from fewer but stronger genetic alterations. Therefore, comparing $\mathrm{CMCL}$ with aMCL is not sufficient for the detection of transformation specific genes because it is not possible to distinguish them morphologically. Alternatively, we expected that using results from the gene selection method in combination with that of iMCL cases $(n=2)$ would provide the candidate genes restricted within aMCL having genetic stepwise process. Additionally, using iMCL samples as a discovery set can compensate for individual differences between cases. For a concrete explanation of transformation, total RNA from whole tumor tissue samples, cMCL $(n=4)$ and aMCL $(n=5)$ were used [Fig. 2(I)]. We also compared classical areas with aggressive areas in iMCL obtained by LMD [Fig. 2(II)]. Finally, we selected the overlapping genes differentially expressed in both comparisons [Fig. 2(III)].

Laser microdissection (LMD). The tissue samples were immediately frozen in acetone/dry ice and stored at $-80^{\circ} \mathrm{C}$ for microdissection. The lymph node samples were embedded in an optical cutting temperature (OCT) compound (Sakura Finetek, Tokyo, Japan) and frozen in liquid nitrogen. Cryosections (10- $\mu$ m-thick) were mounted on $2.0-\mu$ m-thick PEN-Membrane slides (MicroDissect $\mathrm{GmbH}$, Herborn, Germany). After fixation in 100\% ethanol, the slides were stained rapidly with Toluidine Blue O (Chroma-Gesellschaft Schmid GmbH \& Co., Köngen, Germany) and then washed in DEPC-treated water and air-dried with a fan. The frozen sections were microdissected with a Leica LMD6000 laser microdissection system by following the company's protocol (Leica, Wetzlar, Germany). The sorting regions were microdissected from the tissue sections with LMD (Fig. 3), and the dissected cells were collected in $0.5 \mathrm{ml}$ tubes filled with $50 \mu \mathrm{l}$ lysis buffer for RNA extraction.

RNA extraction and biotinylated cRNA amplification. Total RNA was extracted from the LMD-obtained samples with an RNAqueous-Micro kit (Ambion, Austin, TX, USA) according to the manufacturer's instructions. For CMCL and aMCL tissues, total RNA was isolated using TRIzol reagent (Invitrogen, Carlsbad, CA, USA). RNA samples were quantified by an ND-1000 spectrophotometer (NanoDrop Technologies, Wilmington, DE, USA) and the quality was confirmed with an Experion System (Bio-Rad Laboratories, Hercules, CA, USA). Both cRNA amplification and labeling with biotin were used to prepare samples for gene expression profiling by microarray analysis. Briefly, 350-500 ng total RNA was amplified overnight (14 h) with the Illumina Total Prep RNA Amplification kit (Ambion) in accordance with the manufacturer's protocol. Reaction cRNA was biotinylated during in vitro transcription.

Illumina BeadChips microarray. Sentrix Human WG-6 v3.0 Expression BeadChips were purchased from Illumina, Inc. 


\section{Gene selection methods for transformation}

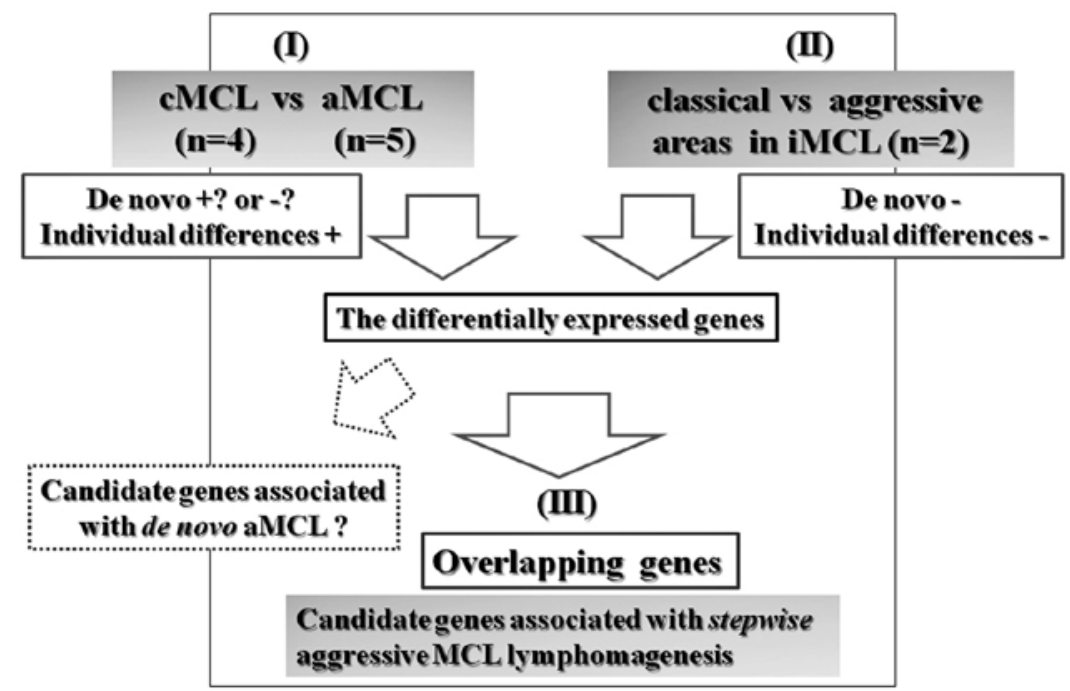

Figure 2. Gene selection methods for the transformation. For the analysis of the method (I), we compared total RNAs from whole tumor tissue samples, cMCL (n=4) with aMCL $(\mathrm{n}=5)$. To compensate for individual differences and the influence of de novo aMCL, we also compared microdissected classical area with the aggressive area in iMCL $(n=2)$ obtained by means of LMD [method (II)]. Finally, we selected the overlapping genes (III) differentially expressed in both comparisons.

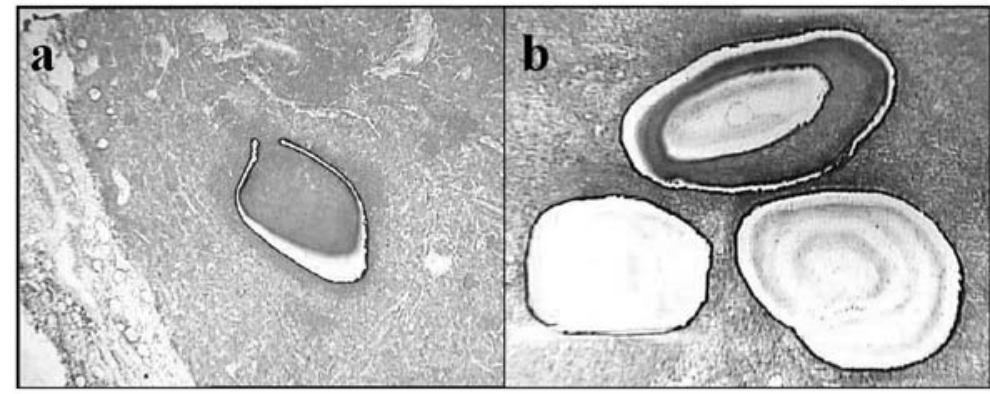

Figure 3. Microdissection of the present cases (3a and 3b). After Toluidine blue staining, a section was subjected to LMD. Figures showing microdissected areas of (a) classical form in iMCL (case no. MCL132) and (b) of tumor cells in MCL in situ (case no. MCL113), respectively.

(San Diego, CA, USA). More than 48,000 different bead types, each with a 50-base gene-specific probe, are represented on a single Beadchip. For each probe represented on the array, beads are assembled with an average 30 -fold redundancy. A hybridization mixture containing $1.5 \mu \mathrm{g}$ biotinylated cRNA was hybridized to the beadchips at $58^{\circ} \mathrm{C}$ overnight $(18 \mathrm{~h})$ before being washed and stained with streptavidin-Cy3 (GE Healthcare, Buckinghamshire, UK) according to the manufacturer's protocol. Beadchips were scanned on Illumina BeadStation 500 and fluorescent hybridization signals were assessed with Illumina BeadStudio software.

Immunohistochemistry. The samples were also evaluated for expression of markers such as Wnt3 (Abcam Inc., Cambridge, MA, USA), phosphorylated- $\beta$-catenin (Ser552) (p $\beta$-catenin-S552) (Cell Signaling, Boston, MA, USA), and Ki-67 (Dako Cytomation, Glostrup, Denmark). Formalin-fixed, paraffin-embedded tissues were used for all immunohistochemical stains. Antibody dilutions and antigen retrieval procedures were performed as standards.
Data analysis and filter criteria. Pre-processing was performed on the raw signal intensities of all samples by $\log _{2}$-transformation and normalization using the quantile algorithm of the 'preprocess Core' library package (8) in Bioconductor (9). We selected the probes (excluding the control probes) where the detection p-value was $<0.01$ in all samples, and used only these probes to identify differentially expressed genes. For the samples meeting the criteria of initiation (MCL in situ vs. normal mantle zone B lymphocytes) and transformation (I) (cMCL vs. aMCL), we applied Linear Models for Microarray Analysis (limma) package (10) of Bioconductor. We used a cutoff of limma p-value $<0.05$ and absolute log-fold change $(\log \mathrm{FCl})>0.5$ to assess differentially expressed genes in each comparison. For the transformation (II) comparison (classical area vs. aggressive area in iMCL), we selected probes that contained a 'P' flag in both iMCL samples. To identify up or downregulated genes between aggressive and classical areas, we calculated Z-scores (11) and ratios (non-log scaled fold change) from the normalized signal intensities of each probe. Then we established criteria for regulated genes: absolute Z-score (IZI) 


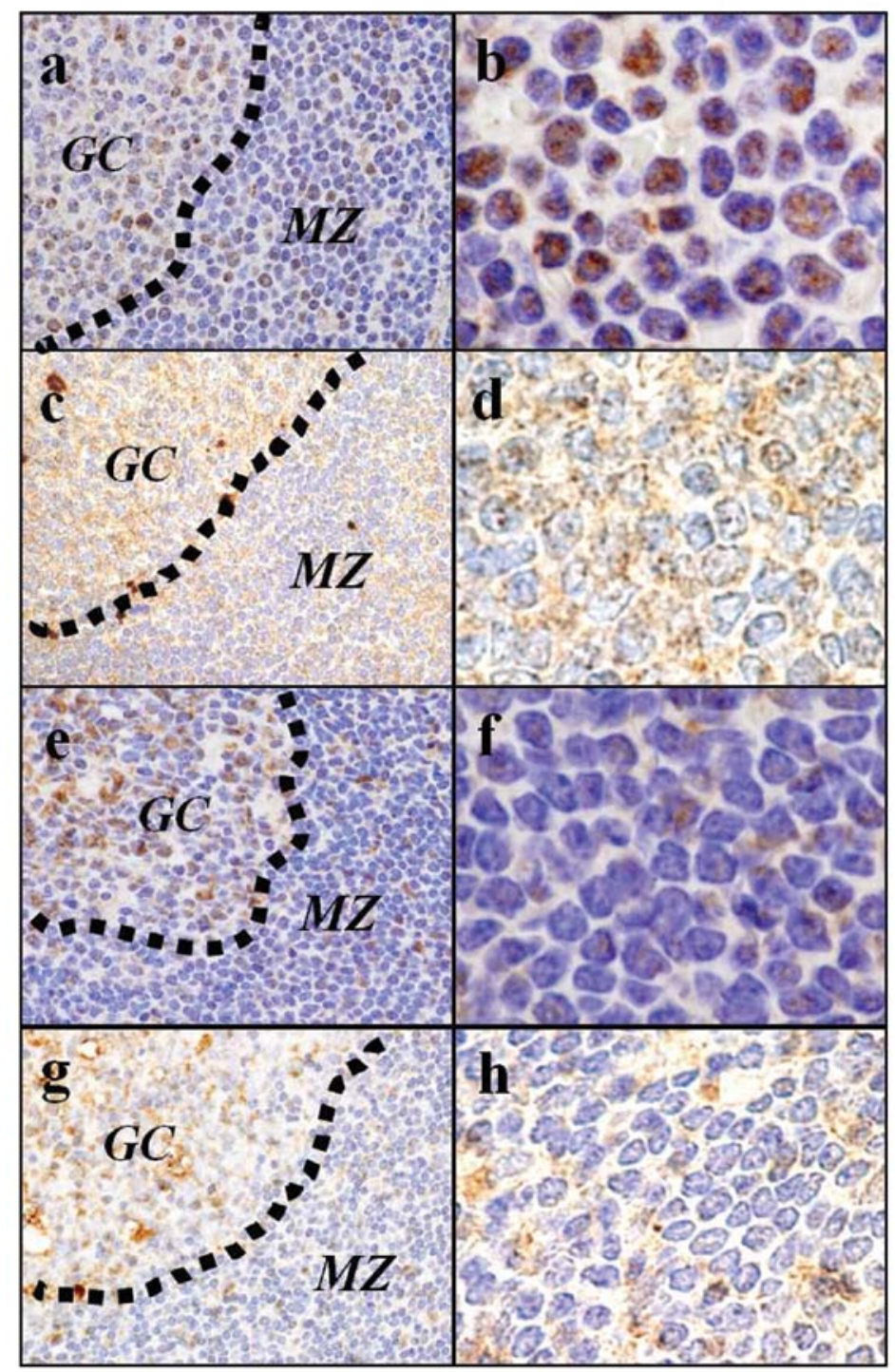

Figure 4. Immunohistochemistry of (a, b, e and f) p $\beta$-catenin-S552 and (c, d, g and h) Wnt3. The immunohistochemical studies revealed that the tumor cells of the MCL in situ showed nuclear localization of p $\beta$-catenin-S552 (a and b) with high levels of cytoplasmic Wnt3 staining (c and d). The reactive mantle zone B cells were negative for $\mathrm{p} \beta$-catenin-S552 in the nuclear (e and f) with low lelels of cytoplasmic Wnt3 staining ( $\mathrm{g}$ and $\mathrm{h}$ ). $\mathrm{p} \beta$-catenin-S552 and Wnt3, original magnification: a, c, e and $\mathrm{g}, \mathrm{x} 1,000$ and b, $\mathrm{d}, \mathrm{f}$ and $\mathrm{h}, \mathrm{x} 2,000$. GC, germinal center; MZ, marginal zone.

$>2$. Ingenuity Pathway Analysis (IPA6.0; Ingenuity Systems, Redwood, CA, USA; http://www.ingenuity.com) was used to identify networks of interacting genes. Lists of expressed (up and downregulated) genes were uploaded for IPA. A heat map of the differentially expressed genes was generated by $\mathrm{MeV}$ software (12). Array data are available on the Gene Expression Omnibus (GEO) website under accession numbers GSE30189 (http://www.ncbi.nlm.nih.gov/geo/).

\section{Results}

Differentially expressed genes in initiaion. In the analysis of MCL initiation, we selected 1,538 genes (851 upregulated and 787 downregulated) which showed significant differences $(\mathrm{p}<0.05)$ between normal mantle zone B lymphocytes and MCL in situ samples (http://www.ncbi.nlm.nih.gov/geo/). The identification of $C C N D 1$ as the most significantly upregulated gene in 'initiation' samples gives us confidence that these array experiments are measuring biologically relevant differences between the sample types. The top 10 up-and down-regulated genes in MCL in situ were: up, CCND1, FCGBP, IL17RB, NULL (probe ID; 5390192), WNT3, D4S234E, FBLN2, CPXM1, DBN1 and TEAD2; down, PLAC8, LOC439949, HVCN1, FGR, LOC651751, IL7R, DEF8, TXNDC8, GZMB and SLAMF1.

For further analysis, we focused on the canonical $\beta$-catenindependent Wnt pathway (WCP), which is critically involved in cell fate and diffrerentiation (13). As reasons for this, IPA analysis revealed that a significant number of differentially expressed genes $(n=23)$ belong to this category $(p=0.016)$, and none of the genes in this pathway were observed as differentially expressed in the transformation experiments (Table II). Whether the cytosolic pool of $\beta$-catenin participates in WCP signaling is dictated by the availability of its binding partners, and these binding interactions are regulated by phosphorylation (14). Phosphorylation of $\beta$-catenin at Ser552 by AKT can 
Table II. Genes associated with Wnt signaling pathway $(\mathrm{p}<0.05)$

Time course $\mathrm{p}<0.05$

\begin{tabular}{|c|c|c|c|c|c|c|}
\hline \multirow{3}{*}{$\begin{array}{l}\text { Acc } \\
\text { NM_053056.2 }\end{array}$} & \multirow{3}{*}{$\begin{array}{l}\text { Gene name } \\
\text { CCND1 }\end{array}$} & \multirow[b]{2}{*}{ Gene description } & \multicolumn{2}{|c|}{ Normal MZ vs. MCL in situ } & \multicolumn{2}{|c|}{ cMCL vs. aMCL } \\
\hline & & & Fold change & P-value & Fold change & P-value \\
\hline & & Cyclin D1 & 4.41 & $2.51 \mathrm{E}-08$ & NS & NS \\
\hline NM_030753.3 & $\mathrm{WNT}^{\mathrm{a}}$ & $\begin{array}{l}\text { Wingless-type MMTV } \\
\text { integration site family, member } 3\end{array}$ & 2.65 & 0.000063 & NS & NS \\
\hline NM_024507.2 & KREMEN2 & $\begin{array}{l}\text { Kringle containing transmembrane } \\
\text { protein } 2\end{array}$ & 1.52 & 0.001595 & NS & NS \\
\hline NM_002335.1 & LRP5 $^{\text {b }}$ & $\begin{array}{l}\text { Low density lipoprotein } \\
\text { receptor-related protein } 5\end{array}$ & 1.04 & 0.000428 & NS & NS \\
\hline NM_020335.1 & VANGL2 & Vang-like 2 (van gogh, Drosophila) & 1.00 & 0.000208 & NS & NS \\
\hline NM_001466.2 & FZD2 & Frizzled homolog 2 (Drosophila) & 0.90 & 0.001185 & NS & NS \\
\hline NM_003199.2 & $\mathrm{TCF}^{\mathrm{b}}$ & Transcription factor 4 & 0.81 & 0.000155 & NS & NS \\
\hline NM_004380.2 & $\mathrm{CREBBP}^{\mathrm{b}}$ & CREB binding protein & 0.72 & 0.000165 & NS & NS \\
\hline NM_001760.2 & GRN & Granulin & 0.67 & 0.014332 & NS & NS \\
\hline NM_138713.2 & NFAT5 & $\begin{array}{l}\text { Nuclear factor of activated T-cells } 5 \text {, } \\
\text { tonicity-responsive }\end{array}$ & 0.57 & 0.001586 & NS & NS \\
\hline NM_002738.5 & PRKCB & Protein kinase $\mathrm{C}$, beta & 0.55 & 0.003660 & NS & NS \\
\hline NM_152221.2 & $\mathrm{CSNK}_{1 \mathrm{E}^{\mathrm{b}}}$ & Casein kinase 1 , epsilon & 0.53 & 0.004865 & NS & NS \\
\hline NM_001006610.1 & SIAH1 & $\begin{array}{l}\text { Seven in absentia homolog } 1 \\
\text { (Drosophila) }\end{array}$ & 0.52 & 0.001216 & NS & NS \\
\hline NM_004423.3 & DVL3 $^{\mathrm{b}}$ & $\begin{array}{l}\text { Dishevelled, dsh homolog } 3 \\
\text { (Drosophila) }\end{array}$ & 0.45 & 0.007706 & NS & NS \\
\hline NM_020248.2 & CTNNBIP1 & Catenin, beta interacting protein 1 & 0.42 & 0.016759 & NS & NS \\
\hline NM_003200.1 & TCF3 & $\begin{array}{l}\text { Transcription factor } 3 \text { (E2A immunoglobulin } \\
\text { enhancer binding factors E12/E47) }\end{array}$ & 0.35 & 0.031493 & NS & NS \\
\hline NM_181492.1 & TCF20 & Transcription factor 20 (AR1) & -0.39 & 0.020984 & NS & NS \\
\hline NM_033120.2 & NKD2 & Naked cuticle homolog 2 (Drosophila) & -0.39 & 0.037969 & NS & NS \\
\hline NM_002872.3 & $\mathrm{RAC2}$ & $\begin{array}{l}\text { Ras-related C3 botulinum toxin substrate } 2 \\
\text { (rho family, small GTP binding protein Rac2) }\end{array}$ & -0.42 & 0.008708 & NS & NS \\
\hline NM_007236.3 & $\mathrm{CHP}$ & Calcium binding protein $\mathrm{P} 22$ & -0.45 & 0.005099 & NS & NS \\
\hline NM_002467.3 & MYC & $\begin{array}{l}\text { V-myc myelocytomatosis viral oncogene } \\
\text { homolog (avian) }\end{array}$ & -0.48 & 0.034874 & NS & NS \\
\hline NM_002737.2 & PRKCA & Protein kinase $\mathrm{C}$, alpha & -0.72 & 0.002025 & NS & NS \\
\hline NM_016269.2 & LEF1 & Lymphoid enhancer-binding factor 1 & -0.87 & 0.004964 & NS & NS \\
\hline
\end{tabular}

${ }^{a} \mathrm{MCL}$ signature genes in agreement with CCND1-negative lymphomas that had MCL morphology and immunophenotype were classified as MCL (17). ${ }^{\mathrm{b}}$ Genes aberrantly expressed in MCL related to WNT signaling pathway compared with naive B cells (6).

enhance $\beta$-catenin/TCF reporter activation, suggesting that $\mathrm{p} \beta$-catenin-S552 is a nuclear-localized form of $\beta$-catenin (active form) (15). Microarray results indicate that $\beta$-catenin was not significantly upregulated in MCL initiation, but immunohistochemical results revealed that the tumor cells of the MCL in situ samples showed nuclear localization of $\mathrm{p} \beta$-catenin-S552 with high levels of cytoplasmic Wnt3 staining (Fig. 4a-d). On the other hand, reactive mantle zone B cells were negative for $\mathrm{p} \beta$-catenin-S552 in the nuclear, and cytoplasmic Wnt3 staining was weak (Fig. 4e-h).

Differentially expressed genes in transformation. With the Illumina BeadStudio software, we detected 710 genes that showed significant differences in expression between cMCL and aMCL groups [Fig. 2(I)] (http://www.ncbi.nlm.nih.gov/ geo/). Gene expression profiling in iMCL showed that 220 genes were differently expressed between microdissected classical area and aggressive area samples [Fig. 2(II)] (http://www.ncbi. nlm.nih.gov/geo/). Finally, we identified 60 overlapping genes [Fig. 2(III)] (Table III), both upregulated $(n=58)$ and downregulated $(n=2)$, which showed significant differences in pre- vs. post-transformation with a $\mathrm{p}$-value of $<0.05$. These genes are visualized as a heat map in Fig. 5.

As may be expected, IPA analysis of the filtered 60 genes revealed that most of these genes $(42 / 60 ; 70 \%)$ were classified in the following categories, all of which are thought to be involved in transformation: 36 genes belonged to the category of 'Cell cycle progression' (UBE2C, BIRC5, CDCA5, TYMS, 


\section{Heat map of the differentially expressed genes in evolutional event}

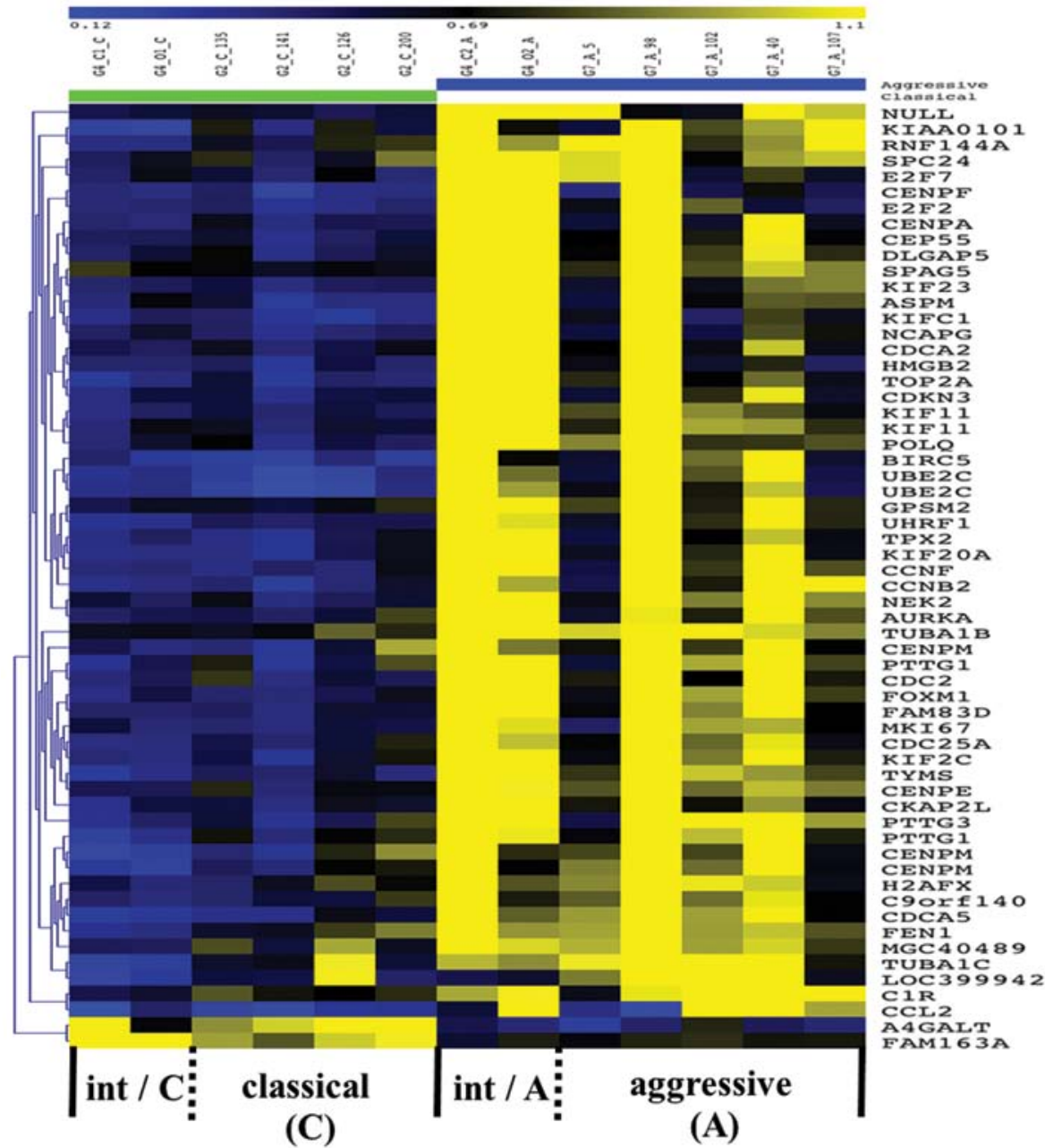

Figure 5. Heat map of the differentially expressed genes in the transformation. The genes displayed in this figure were identified by our selection methods. Color intensity represents the magnitude of deviation from the median. Yellow represents relative expression levels higher than the median level across all tissues and blue represents lower expression levels. The levels of most genes are more strongly upregulated in aggressive compared to classical MCL samples.

KIFC1, TOP2A, FOXM1, CCNF, E2F2, KIF2C, TPX2, KIF11, CDC2, CENPF, NEK2, CDKN3, PTTG1, DLGAP5, CENPE, CDC25A, AURKA, CCNB2, ASPM, KIF20A, UHRF1, CEP55, NCAPG, MKI67, KIF23, H2AFX, GPSM2); 28 'DNA replication, recombination and repair' genes (AURKA, BIRC5, CENPE, DLGAP5, KIF2C, KIFC1, NCAPG, TOP2A, CCNB2, GPSM2, NEK2, PTTG1, KIF11, TPX2, CDCA5, CDK1, CDC25A, E2F2, FEN1, FOXM1, HMGB2, KIAA0101, H2AFX, POLQ, TYMS, KIF23, CENPA, UHRF1); 10 'cell death' genes (A4GALT, AURKA, BIRC5, CCL2, CDC25A, CDCA2, CDK1, E2F2, NEK2, PTTG1, TOP2A, TYMS, $U B E 2 C, C E N P F, F E N 1, F O X M 1, H M G B 2, K I F 2 C$ ). As seen in Fig. 6, these genes recapitulate much of the p53 interaction network, a network centrally involved in cell cycle progression. Most of these genes (AURKA, BIRC5, CDC2, CDC25A, CENPF, CENPE, CCNB2, FOXM1, NEK2, PTTG1, TPX2, $A S P M, T O P 2 A, D L G A P 5, K I F 2 C, K I F 23, U B E 2 C)$ were considered important players in MCL transformation because of their known function as mitotic regulators, which mediate cell cycle progression during the $\mathrm{G} 2 / \mathrm{M}$ transition. $C D C 2$, FOXMI and BIRC5 in particular interact with many other highly expressed genes in aMCL, which suggests that they may play a critical role in transformational events along with $p 53$. Of these transformation-associated genes, only CDCA5 and MKI67 were also significantly changed in initiation, while the remaining 58 genes were aberrantly expressed specifically in transformation.

\section{Discussion}

Biological heterogeneity has become an important position in the current understanding of MCL. To meet this need, we performed cDNA microarray experiments stratifying MCL samples into four morphological grades based on the files including a large series of patients. In order to investigate the molecular mechanisms underlying these differences, we compared initial component samples or transformational morphology samples and identified genes associated with the Wnt signaling pathway and several known mitotic regulators as differentially regulated.

Cell cycle alterations resulting in unscheduled proliferation are strongly associated with the evolution of malignant tumors. 
Table III. Genes associated with the development of aggressive form of MCL (cMCL vs. aMCL using by iMCL).

\begin{tabular}{|c|c|c|c|c|}
\hline Acc & Gene name & Gene description & Fold change & P-value \\
\hline NM_181800.1 & UBE2C & Ubiquitin-conjugating enzyme E2C & 1.84 & 0.000371 \\
\hline NM_181803.1 & UBE2C & Ubiquitin-conjugating enzyme E2C & 1.74 & 0.000558 \\
\hline NM_002982.3 & CCL2 & Chemokine (C-C motif) ligand 2 & 1.60 & 0.038047 \\
\hline NM_001168.2 & BIRC5 $5^{\mathrm{d}, \mathrm{e}}$ & Baculoviral IAP repeat-containing 5 & 1.58 & 0.000489 \\
\hline NM_080668.2 & CDCA5 & Cell division cycle associated 5 & 1.27 & 0.003079 \\
\hline NM_004701.2 & $\mathrm{CCNB} 2^{\mathrm{c}}$ & Cyclin B2 & 1.21 & 0.004265 \\
\hline NM_001071.1 & TYMS $^{\mathrm{c}}$ & Thymidylate synthetase & 1.11 & 0.001068 \\
\hline NM_002263.2 & KIFC1 & Kinesin family member $\mathrm{C} 1$ & 1.09 & 0.007440 \\
\hline NM_001067.2 & TOP2A ${ }^{\mathrm{a}, \mathrm{c}}$ & Topoisomerase (DNA) II alpha $170 \mathrm{kDa}$ & 1.08 & 0.006076 \\
\hline NR_002734.1 & PTTG3 & Pituitary tumor-transforming 3 & 1.07 & 0.014845 \\
\hline NM_018136.3 & $\mathrm{ASPM}^{\mathrm{b}, \mathrm{c}, \mathrm{d}, \mathrm{e}}$ & $\begin{array}{l}\text { 'Abnormal spindle (ASP) homolog, } \\
\text { microcephaly associated (Drosophila)' }\end{array}$ & 1.07 & 0.005186 \\
\hline NM_021953.2 & $\mathrm{FOXM}^{\mathrm{c}}$ & Forkhead box M1 & 1.01 & 0.003420 \\
\hline NM_005733.1 & $\mathrm{KIF} 20 \mathrm{~A}^{\mathrm{c}}$ & Kinesin family member $20 \mathrm{~A}$ & 0.99 & 0.010183 \\
\hline NM_001761.1 & $\mathrm{CCNF}$ & Cyclin F & 0.99 & 0.006634 \\
\hline NM_001048201.1 & $\mathrm{UHRF}^{\mathrm{a}, \mathrm{e}}$ & Ubiquitin-like with PHD and ring finger domains 1 & 0.97 & 0.007291 \\
\hline NM_001002876.1 & CENPM & Centromere protein $\mathrm{M}$ & 0.94 & 0.016725 \\
\hline NM_004091.2 & $\mathrm{E} 2 \mathrm{~F} 2$ & E2F transcription factor 2 & 0.94 & 0.019567 \\
\hline NM_001002876.1 & CENPM & Centromere protein $\mathrm{M}$ & 0.94 & 0.035235 \\
\hline NM_030919.2 & FAM83D & 'Family with sequence similarity 83, member D' & 0.92 & 0.007561 \\
\hline NM_024053.3 & CENPM & Centromere protein $\mathrm{M}$ & 0.92 & 0.046319 \\
\hline NM_006845.2 & $\mathrm{KIF} 2 \mathrm{C}$ & Kinesin family member $2 \mathrm{C}$ & 0.90 & 0.016135 \\
\hline NM_199420.3 & POLQ & 'Polymerase (DNA directed), theta' & 0.89 & 0.004755 \\
\hline NM_012112.4 & TPX2 & $\begin{array}{l}\text { 'TPX2, microtubule-associated, } \\
\text { homolog (Xenopus laevis)' }\end{array}$ & 0.89 & 0.016590 \\
\hline NM_018131.3 & CEP55 & Centrosomal protein $55 \mathrm{kDa}$ & 0.88 & 0.006728 \\
\hline NM_004523.2 & $\mathrm{KIF} 11^{\mathrm{c}}$ & Kinesin family member 11 & 0.88 & 0.005905 \\
\hline NM_022346.3 & NCAPG & 'Non-SMC condensin I complex, subunit G' & 0.88 & 0.014421 \\
\hline XM_934471.1 & LOC399942 & - & 0.87 & 0.039823 \\
\hline NM_002417.3 & MKI67e & Antigen identified by monoclonal antibody Ki-67 & 0.86 & 0.022646 \\
\hline NM_001786.2 & $\mathrm{CDC} 2^{\mathrm{b}, \mathrm{c}, \mathrm{d}, \mathrm{e}}$ & 'Cell division cycle $2, \mathrm{G} 1$ to $\mathrm{S}$ and $\mathrm{G} 2$ to $\mathrm{M}$ ' & 0.86 & 0.023818 \\
\hline NM_016343.3 & $\mathrm{CENPF}^{\mathrm{b}, \mathrm{c}, \mathrm{e}}$ & 'Centromere protein F, 350/400ka (mitosin)' & 0.84 & 0.032315 \\
\hline NM_002497.2 & $\mathrm{NEK}^{\mathrm{c}}$ & NIMA (never in mitosis gene a)-related kinase 2 & 0.83 & 0.002697 \\
\hline NM_178448.2 & C9orf140 & Chromosome 9 open reading frame 140 & 0.81 & 0.023110 \\
\hline NM_004219.2 & PTTG1 & Pituitary tumor-transforming 1 & 0.80 & 0.047544 \\
\hline NM_004856.4 & $\mathrm{KIF} 23^{\mathrm{c}, \mathrm{e}}$ & Kinesin family member 23 & 0.80 & 0.007960 \\
\hline NM_005192.2 & $\mathrm{CDKN}^{\mathrm{a}, \mathrm{c}}$ & Cyclin-dependent kinase inhibitor 3 & 0.79 & 0.029828 \\
\hline NM_004219.2 & PTTG1 & Pituitary tumor-transforming 1 & 0.79 & 0.038189 \\
\hline NM_002129.2 & $\mathrm{HMGB}^{\mathrm{a}, \mathrm{c}}$ & High-mobility group box 2 & 0.79 & 0.045729 \\
\hline NM_152515.2 & CKAP2L & Cytoskeleton associated protein 2-like & 0.78 & 0.036949 \\
\hline NM_004523.2 & $\mathrm{KIF} 11^{\mathrm{c}}$ & Kinesin family member 11 & 0.78 & 0.004380 \\
\hline NM_014746.2 & RNF144A & Ring finger protein 144A & 0.78 & 0.005460 \\
\hline NM_014750.3 & DLGAP5 & $\begin{array}{l}\text { 'Discs, large (Drosophila) } \\
\text { homolog-associated protein 5' }\end{array}$ & 0.76 & 0.009518 \\
\hline NM_014736.4 & KIAA0101 & KIAA0101 & 0.76 & 0.045005 \\
\hline NM_032704.2 & TUBA1C & 'Tubulin, alpha 1c' & 0.73 & 0.027816 \\
\hline NM_001813.2 & $\mathrm{CENPE}^{\mathrm{c}, \mathrm{d}}$ & 'Centromere protein E, $312 \mathrm{kDa}$ ' & 0.73 & 0.013906 \\
\hline ВC004287 & NULL & Null & 0.73 & 0.019170 \\
\hline NM_001789.2 & CDC25A & Cell division cycle 25 homolog A (S. pombe) & 0.73 & 0.025460 \\
\hline NM_002105.2 & $\mathrm{H} 2 \mathrm{AFX}$ & 'H2A histone family, member X' & 0.72 & 0.038540 \\
\hline NM_203394.2 & $\mathrm{E} 2 \mathrm{~F} 7$ & E2F transcription factor 7 & 0.68 & 0.032400 \\
\hline NM_001042426.1 & CENPA $^{\mathrm{c}}$ & Centromere protein $\mathrm{A}$ & 0.65 & 0.044459 \\
\hline NM_198434.1 & AURKA $^{c}$ & Aurora kinase A & 0.62 & 0.042391 \\
\hline
\end{tabular}


Table III. Continued.

\begin{tabular}{lllr}
\hline Acc & Gene name & \multicolumn{1}{c}{ Gene description } & Fold change \\
\hline NM_152562.2 & CDCA2 & Cell division cycle associated 2 & 0.61 \\
NM_006082.2 & TUBA1B ${ }^{\text {a,f }}$ & 'Tubulin, alpha 1b' & 0.61 \\
NM_004111.4 & FEN1 & Flap structure-specific endonuclease 1 & 0.002775 \\
NM_013296.3 & GPSM2 & 'G-protein signaling modulator 2 & 0.60 \\
& & (AGS3-like, C. elegans)' & 0.55 \\
NM_182513.1 & SPC24 & 'SPC24, NDC80 kinetochore complex component, & 0.035752 \\
& & homolog (S. cerevisiae) & 0.55 \\
XR_016048.1 & MGC40489 & - & 0.034394 \\
NM_001733.4 & C1R & 'Complement component 1, R subcomponent' & 0.53 \\
NM_006461.3 & SPAG5' & Sperm associated antigen 5 & 0.52 \\
NM_173509.2 & FAM163A & 'Family with sequence similarity 163, member A' & 0.049409 \\
NM_017436.4 & A4GALT & 'Alpha 1,4-galactosyltransferase' & -0.39 \\
\hline
\end{tabular}

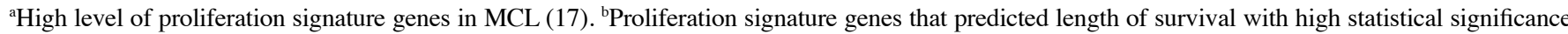
in MCL (17). 'Identification of genes diffferentally expressed in MCL with low levels of long CCND1 transcript compared to other MCL (fold up) (18). ${ }^{\mathrm{d}}$ Survival prediction genes in MCL (20). ${ }^{\mathrm{e}}$ Most significant genes separating good and poor prognosis in MCL (fold up) (20). ${ }^{\mathrm{f}}$ Genes separating good and poor prognosis not associated with cell cycle (fold up) (20).

\section{Gene network generated through the use of IPA}

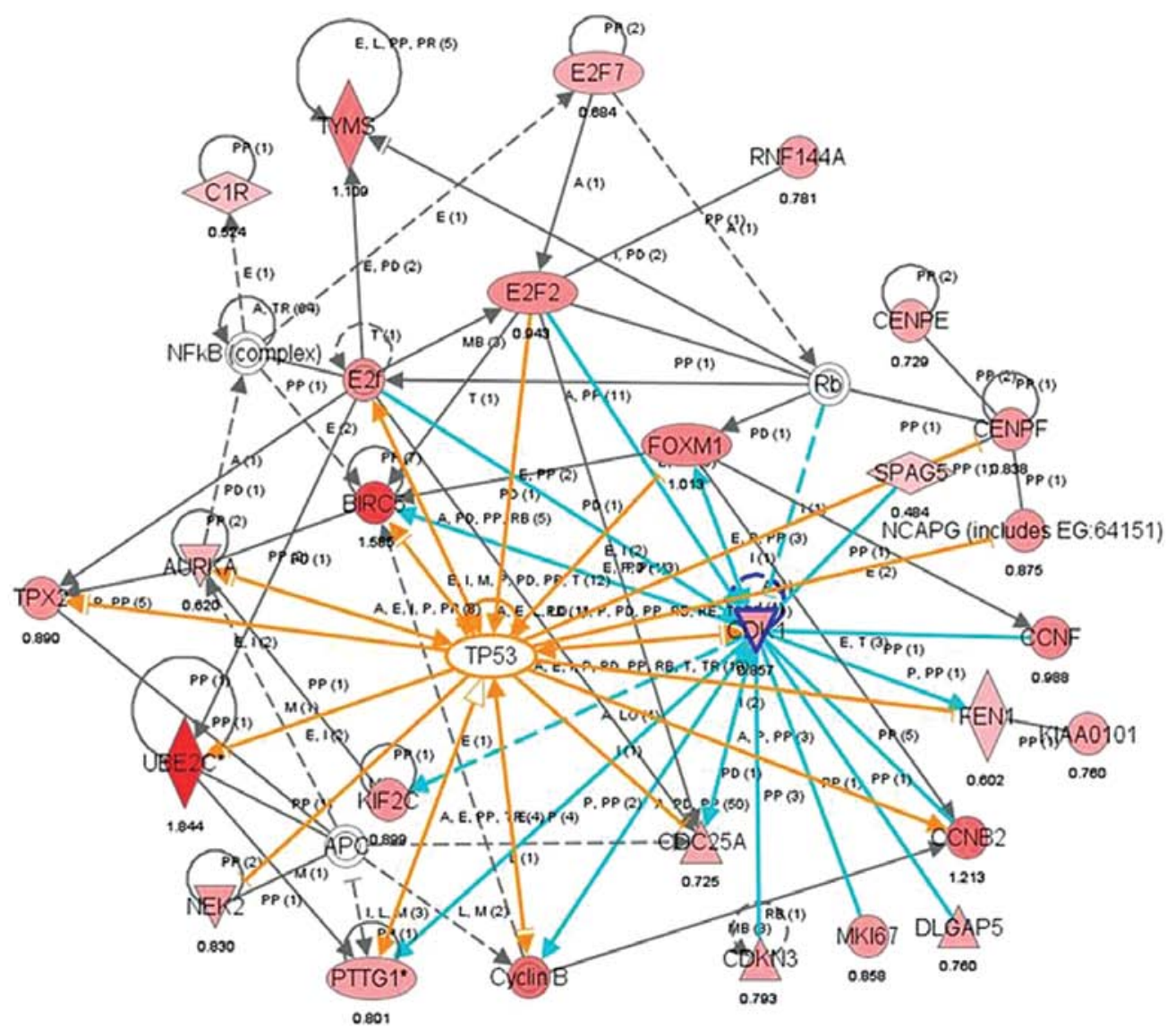

Figure 6. Gene network generated through the use of IPA. IPA was applied to 60 genes obtained by using iMCL as the transformation to create a network interrelated with mediator genes. Interacting nodes are defined by either direct relationships, which require direct physical contact (solid arrows), or indirect relationships (dashed arrows). The direction of the arrows shows the direction of the interaction. The chart clearly and in detail showed the network composed of p53 and several of its interaction partners. Most of these are mitotic regulators, and CDC2, FOXM1 and BIRC5 in particular interact with many other highly expressed genes in $\mathrm{aMCL}$. The blue and orange lines indicate the connection with CDK1 (CDC2) and TP53, respectively. Red molecules indicate higher expression in aMCL than in $\mathrm{CMCL}$ at gene level. 
Most of these changes alter pathways involved in G1 progression or the G1/S transition (16). With regard to MCL, the 'Lymphoma and Leukemia Molecular Profiling Project (LLMPP)' demonstrated that the length of survival of MCL patients depends upon quantitative differences in progression from G1/S phase of the cell cycle (17). Sander et al also showed that a subset of MCL tumors with low levels of the long CCNDI transcript is highly proliferative and some of its related genes have homology to the group of cell cycle (G1/S) promoting $E 2 F$ transcription partners (18). Indeed, there is a strong correlation between these reported genes and the 60 we identified in this study as involved in transformation (Table III), which emphasizes the importance of G1/S regulators in MCL evolution. The morphological classifications used in this study were based on our previous findings that MCL presents in three morphological evolutions: classical, intermediate and aggressive, and that the aggressive form is considered an important clinical signal of poor prognosis (1). Therefore, it might be natural to expect that dysregulation of G1/S proliferation signature genes is necessary to determine both the length of MCL patient survival and the time to neoplasm transformation.

Although the dysregulation of progression through mitosis does not directly promote proliferation, a few centrosomal and mitotic proteins (such as AURKA, PLK1 and PTTG1) have been reported to act as oncogenes (19). Interestingly, our results highlight a large number of mitotic regulators $(C D C 2$, AURKA, BIRC5, CDC25A, CENPE, CCNB2, FOXM1, NEK2, PTTG1, TPX2, DLGAP5, KIF2C, KIF23 and UBE2C) in addition to $\mathrm{G} 1 / \mathrm{S}$ regulators $(C D C 25 A, E 2 F 2, F O X M 1$ and TYMS). Of these genes, CDC2, FOXM1 and BIRC5 interact with many other highly expressed mitotic regulators within the $p 53$ interaction network, suggesting that specific mitotic regulators facilitate the transformation of MCL into more aggressive form. In support of this hypothesis, Blenk et al identified significantly differentially expressed genes between samples with good and poor prognosis in MCL using exploratory analysis of gene expression values and CGH data (20). Surprisingly, the majority of the genes highlighted in that study (BIRC5, ASPM, MKI67, UHRF1, CDC2, CENPF and $K I F 23)$, including their survival predictor of genes for MCL patients (CENPE, CDC2, BIRC5 and ASPM), overlap with our current findings (Table III). These observations imply that both $C D C 2$ and BIRC5 expression play central roles in MCL transformation and thus reflect prognosis.

$C D C 2$ (also known as $C D K 1$ ) is one of the master regulators of mitosis, as it is involved in the centrosome cycle and early mitotic events. Hui et al reported that elevated protein levels of CDC2 are correlated with the expression of proliferation marker, and represent a useful and simple method in evaluating the prognosis of MCL patients (21). Although BIRC5 (also known as survivin) is known as a member of inhibitor of apoptosis proteins gene family (22), several studies have shown that its role in cancer is not limited to apoptosis inhibition (23). Accordingly, BIRC5 expression levels are higher in aMCL samples than cMCL samples and are associated with proliferative activity and survival of the patients (24).

The role of FOXM1 in lymphoma has not been reported in the literature. However, FOXM1 was also significantly upregulated genes in MCL transformation $(\mathrm{FC} ; 1.01$ and $\mathrm{p}<0.005)$ in our results. FOXM1 is a typical proliferation-associated transcription factor, and is intimately involved in mitosis regulation (25). Namely, FOXM1 directly or indirectly (via $M Y C$ ) regulates genes that control G1/S transition, S-phase progression, G2/M transition and $\mathrm{M}$-phase progression (25), supporting that it is involved in tumorigenesis. It is of interest that its expression level is known to be increased in several other tumor grades, such as prostate carcinoma and glioblastoma $(26,27)$. Thus, all of these genes, as well as the other mitotic regulators we found to have differential expression in MCL transformation, are potentially attractive therapeutic targets or strong diagnostic tools.

Alterations in the DNA damage response pathway and mitotic checkpoints through $p 53$ are major additional genetic events in MCL, as indicated by the high rate of tetraploidy found in aMCL (28). In fact, p53 inactivation in MCL as a consequence of deletion or mutation occurs more frequently in the aggressive form than in the classic form (29). However, inactivating mutations of p53 are found in only $38 \%$ of aMCL cases $(30,31)$, suggesting that several other genetic alterations also contribute to inactivation of the $p 53$ pathway. Currently, ATM deletions are thought to be strongly associated with the dysregulation of the DNA damage response pathway through $p 53$, and are probably present in the early phase of MCL (17,32-34). Our microarray experiments have revealed that $A T M$ was significantly downregulated in initiation with strong fold change $(\mathrm{FC}=-0.73)$, while there was no significant change observed in transformation. In the results of our previous study using immunohistochemistry, higher cell positivity of p53 (DO7) was observed in iMCL than in cMCL, while there was no significant difference between iMCL and aMCL (1). This is due to the fact that there is a high concordance between p53 (DO7) nuclear overexpression and gene mutation in human carcinomas (35). These results strongly indicated that dysregulation of the $A T M / p 53$ pathway in MCL would occur at undetectable levels as a relatively early phenomenon. After that, the disruption of central mitotic regulators ( $C D C 2, B I R C 5$ and $F O X M 1)$ is responsible for the induction of chromosomal instability. Combined, these factors play an important pathogenic role in the evolution of MCL, perturbing the regulation of tumor cell cycling at the $\mathrm{G} 2 / \mathrm{M}$ transition.

The mechanisms of the initial lymphomagenesis of MCL in addition to the $I g H / C C N D I$ remain unclear, and new approaches are urgently needed to elucidate which genes and signaling pathways contribute to this event. Our results support the hypothesis that aberrant Wnt3 signaling is required for the MCL lymphomagenesis, because a significant number of WCP associated genes were aberrantly expressed in the initiation and not significantly changed in the transformation. Moreover, immunohistochemical findings revealed the special activation of WCP.

The significance of WCP signaling in tumor initiation may be straightforward from the view point of the adenoma-carcinoma sequence (36). Deletion of the $A P C$ gene resulting in the activation of WCP is a consistent finding among the earliest events in both de novo and sporadic colon carcinomas. Also, several studies support the notion that WCP can influence both lymphopoiesis $(37,38)$ and hematological malignancies (39). In our initiation experiments, especially Wnt3 and LRP5 were genes with a high average increase (2.65- and 1.04-fold, 
respectively), and are reportedly highly expressed in MCL cases (40).

The binding of Wnt proteins to their respective cell surface receptors, including seven of the transmembrane fizzled $(\mathrm{Fz})$ receptors and low-density lipoprotein receptorrelated protein (LRP5 or 6), activates disheveled (Dvl). Activated Dvl can inhibit the degradation of $\beta$-catenin by the destruction complex, which is composed of adenomatous polyposis coli $(A P C)$, axin, casein kinase $1(C K 1)$ and $G S K-3 \beta$. Consequently, accumulation of $\beta$-catenin in the nucleus regulates gene expression in cooperation with $T$ cell factor (TCF)/lymphocyte enhancer factor ( $L E F)$ transcription factors, resulting in the activation of the WCP target genes such as CCNDI and $c-M y c$ (13).

Using gene expression profiling, Rosenwald et al identified MCL signature genes in CCND1-negative lymphoma cases classified as MCL by both morphology and IHC. Wnt3 is one of these MCL signature genes, suggesting that Wnt3 is more intrinsic to MCL than CCND1 (17). According to Gelebart et al, Wnt 3 is highly and consistently expressed in MCL as detected by WCP-specific oligonucleotide arrays (40). Lako et al showed that Wnt3 protein can enhance haematopoietic commitment during in vitro differentiation of embryonic stem cells (41). From these studies, we speculate that aberrant expression of Wnt3 can emerge as potential activator of lymphomagenesis in MCL because CCNDI itself is an oncogenic target of activated WCP. In addition, several studies have shown that Wnt 3 is highly expressed in B cell CLL $(42,43)$ as well as in MCL.

To summarize, this study shed light on the mechanisms of initiation and evolution in MCL. The resulting patterns of gene dysregulation in these evens strongly indicate that the Wnt signaling pathway plays a critical role in initial lymphomagenesis, and that specific mitotic regulators facilitate transformation into more aggressive forms. Our unique approach may contribute to future understanding of various mature B cell lymphomas. These data hint at a novel system for the classification of low-grade B cell neoplasms using the expression levels of WCP genes and specific mitotic regulator genes as markers for disease stage and predicted outcomes.

\section{Acknowledgements}

The authors would like to thank Konomi Takasu, Mayumi Miura and Kanoko Miyazaki for their technical support.

\section{References}

1. Kimura Y, Sato K, Arakawa F, et al: Mantle cell lymphoma shows three morphological evolutions of classical, intermediate, and aggressive forms, which occur in parallel with increased labeling index of cyclin D1 and Ki-67. Cancer Sci 101: 806-814, 2010.

2. Kaleem Z, Wakoff AR, Smith RP and Hess JL: Blastic transformation of mantle cell lymphoma. Arch Pathol Lab Med 120 577-580, 1996.

3. Bodrug SE, Warner BJ, Bath ML, Lindeman GJ, Harris AW and Adams JM: Cyclin D1 transgene impedes lymphocyte maturation and collaborates in lymphomagenesis with the myc gene. EMBO J 13: 2124-2130, 1994.

4. Hirt C, Schuler F, Dolken L, Schmidt CA and Dolken G: Low prevalence of circulating $\mathrm{t}(11 ; 14)(\mathrm{q} 13 ; \mathrm{q} 32)$-positive cells in the peripheral blood of healthy individuals as detected by real-time quantitative PCR. Blood 104: 904-905, 2004.
5. De Vos S, Krug U, Hofmann WK, et al: Cell cycle alterations in the blastoid variant of mantle cell lymphoma (MCL-BV) as detected by gene expression profiling of mantle cell lymphoma (MCL) and MCL-BV. Diagn Mol Pathol 12: 35-43, 2003.

6. Rizzatti EG, Falcao RP, Panepucci RA, et al: Gene expression profiling of mantle cell lymphoma cells reveals aberrant expression of genes from the PI3K-AKT, WNT and TGFbeta signalling pathways. Br J Haematol 130: 516-526, 2005.

7. Martinez N, Camacho FI, Algara P, et al: The molecular signature of mantle cell lymphoma reveals multiple signals favoring cell survival. Cancer Res 63: 8226-8232, 2003.

8. Bolstad BM, Irizarry RA, Astrand M and Speed TP: A comparison of normalization methods for high density oligonucleotide array data based on variance and bias. Bioinformatics 19: 185-193, 2003.

9. Gentleman RC, Carey VJ, Bates DM, et al: Bioconductor: open software development for computational biology and bioinformatics. Genome Biol 5: R80, 2004.

10. Smyth GK, Michaud J and Scott HS: Use of within-array replicate spots for assessing differential expression in microarray experiments. Bioinformatics 21: 2067-2075, 2005.

11. Quackenbush J: Microarray data normalization and transformation. Nat Genet 32 (Suppl): 496-501, 2002.

12. Saeed AI, Sharov V, White J, et al: TM4: a free, open-source system for microarray data management and analysis. Biotechniques 34: 374-378, 2003.

13. Aguilera O, Munoz A, Esteller M and Fraga MF: Epigenetic alterations of the Wnt/beta-catenin pathway in human disease. Endocr Metab Immune Disord Drug Targets 7: 13-21, 2007.

14. Daugherty RL and Gottardi CJ: Phospho-regulation of beta-catenin adhesion and signaling functions. Physiology (Bethesda) 22: 303-309, 2007.

15. Fang D, Hawke D, Zheng Y, et al: Phosphorylation of beta-catenin by AKT promotes beta-catenin transcriptional activity. J Biol Chem 282: 11221-11229, 2007.

16. Massague J: G1 cell-cycle control and cancer. Nature 432: 298-306, 2004.

17. Rosenwald A, Wright G, Wiestner A, et al: The proliferation gene expression signature is a quantitative integrator of oncogenic events that predicts survival in mantle cell lymphoma. Cancer Cell 3: 185-197, 2003

18. Sander B, Flygare J, Porwit-Macdonald A, et al: Mantle cell lymphomas with low levels of cyclin D1 long mRNA transcripts are highly proliferative and can be discriminated by elevated cyclin A2 and cyclin B1. Int J Cancer 117: 418-430, 2005.

19. Perez de Castro I, de Carcer G and Malumbres M: A census of mitotic cancer genes: new insights into tumor cell biology and cancer therapy. Carcinogenesis 28: 899-912, 2007.

20. Blenk S, Engelmann JC, Pinkert S, et al: Explorative data analysis of MCL reveals gene expression networks implicated in survival and prognosis supported by explorative $\mathrm{CGH}$ analysis. BMC Cancer 8: 106, 2008.

21. Hui D, Reiman T, Hanson J, et al: Immunohistochemical detection of cdc2 is useful in predicting survival in patients with mantle cell lymphoma. Mod Pathol 18: 1223-1231, 2005.

22. Schimmer AD: Inhibitor of apoptosis proteins: translating basic knowledge into clinical practice. Cancer Res 64: 7183-7190, 2004.

23. Mita AC, Mita MM, Nawrocki ST and Giles FJ: Survivin: key regulator of mitosis and apoptosis and novel target for cancer therapeutics. Clin Cancer Res 14: 5000-5005, 2008.

24. Martinez A, Bellosillo B, Bosch F, et al: Nuclear survivin expression in mantle cell lymphoma is associated with cell proliferation and survival. Am J Pathol 164: 501-510, 2004.

25. Wierstra I and Alves J: FOXM1, a typical proliferation-associated transcription factor. Biol Chem 388: 1257-1274, 2007.

26. Liu M, Dai B, Kang SH, et al: FoxM1B is overexpressed in human glioblastomas and critically regulates the tumorigenicity of glioma cells. Cancer Res 66: 3593-3602, 2006.

27. Kalin TV, Wang IC, Ackerson TJ, et al: Increased levels of the FoxM1 transcription factor accelerate development and progression of prostate carcinomas in both TRAMP and LADY transgenic mice. Cancer Res 66: 1712-1720, 2006.

28. Ott G, Kalla J, Ott MM, et al: Blastoid variants of mantle cell lymphoma: frequent bcl-1 rearrangements at the major translocation cluster region and tetraploid chromosome clones. Blood 89: 1421-1429, 1997.

29. Pinyol M, Hernandez L, Cazorla M, et al: Deletions and loss of expression of p16INK4a and p21Waf1 genes are associated with aggressive variants of mantle cell lymphomas. Blood 89: 272-280, 1997. 
30. Hernandez L, Fest T, Cazorla M, et al: p53 gene mutations and protein overexpression are associated with aggressive variants of mantle cell lymphomas. Blood 87: 3351-3359, 1996.

31. Greiner TC, Moynihan MJ, Chan WC, et al: p53 mutations in mantle cell lymphoma are associated with variant cytology and predict a poor prognosis. Blood 87: 4302-4310, 1996.

32. Bentz M, Plesch A, Bullinger L, et al: $\mathrm{t}(11 ; 14)$-positive mantle cell lymphomas exhibit complex karyotypes and share similarities with B-cell chronic lymphocytic leukemia. Genes Chromosomes Cancer 27: 285-294, 2000.

33. Monni O, Oinonen R, Elonen E, et al: Gain of $3 q$ and deletion of $11 \mathrm{q} 22$ are frequent aberrations in mantle cell lymphoma. Genes Chromosomes Cancer 21: 298-307, 1998.

34. Stilgenbauer S, Winkler D, Ott G, et al: Molecular characterization of $11 \mathrm{q}$ deletions points to a pathogenic role of the ATM gene in mantle cell lymphoma. Blood 94: 3262-3264, 1999.

35. Soong R, Robbins PD, Dix BR, et al: Concordance between p53 protein overexpression and gene mutation in a large series of common human carcinomas. Hum Pathol 27: 1050-1055, 1996.

36. Vogelstein B, Fearon ER, Hamilton SR, et al: Genetic alterations during colorectal-tumor development. N Engl J Med 319: $525-532,1988$.
37. Reya T, O'Riordan M, Okamura R, et al: Wnt signaling regulates B lymphocyte proliferation through a LEF-1 dependent mechanism. Immunity 13: 15-24, 2000.

38. Staal FJ and Sen JM: The canonical Wnt signaling pathway plays an important role in lymphopoiesis and hematopoiesis. Eur J Immunol 38: 1788-1794, 2008.

39. Ge X and Wang X: Role of Wnt canonical pathway in hematological malignancies. J Hematol Oncol 3: 33, 2010.

40. Gelebart P, Anand M, Armanious H, et al: Constitutive activation of the Wnt canonical pathway in mantle cell lymphoma. Blood 112: 5171-5179, 2008.

41. Lako M, Lindsay S, Lincoln J, Cairns PM, Armstrong L and Hole N: Characterisation of Wnt gene expression during the differentiation of murine embryonic stem cells in vitro: role of Wnt3 in enhancing haematopoietic differentiation. Mech Dev 103: 49-59, 2001.

42. Lu D, Zhao Y, Tawatao R, et al: Activation of the Wnt signaling pathway in chronic lymphocytic leukemia. Proc Natl Acad Sci USA 101: 3118-3123, 2004.

43. Rosenwald A, Alizadeh AA, Widhopf G, et al: Relation of gene expression phenotype to immunoglobulin mutation genotype in B cell chronic lymphocytic leukemia. J Exp Med 194: 1639-1647, 2001 